CLAWAR 2020: $23^{\text {rd }}$ International Conference on

Climbing and Walking Robots and the Support

Technologies for Mobile Machines,

Moscow, Russian Federation, 24-26 August 2020.

https://doi.org/10.13180/clawar.2020.24-26.08.27

\title{
SIMULATION OF SPACECRAFT BERTHING WITH A ROBOTIC ARM
}

\author{
YU. F. GOLUBEV \\ *Moscow, Keldysh Institute of Applied Mathematics (Russian Academy of Sciences) \\ E-mail: golubev@keldysh.ru
}

A. V. YASKEVICH

Korolev, RSC Energia

E-mail: Andrey.Yaskevich@rsce.ru

\begin{abstract}
The paper presents a method of hybrid simulation used for testing spacecraft berthing with a space robotic arm. The dynamics of the robotic arm and the relative motion of mating objects are calculated with the use of a specially developed math model, with real docking assemblies. Relative motion of these assemblies is reproduced on a computer controlled Six Degree of Freedom facility (6-DOF).
\end{abstract}

\section{Introduction}

On-orbit spacecraft berthing with a robotic arm is implemented when one of the mating spacecraft is delivered to the other as a payload, or when mating involves simple devices that do not ensure a compensation of relatively big misses and damping of approach energy. The robotic arm mounted on one the spacecraft implements rendezvous system functions. The guidance accuracy necessary to form a primary mechanical link is achieved by involving a human operator in the robotic arm control loop.

For a ground testing of such operations, a relevant challenge is to adequately take into account spatial dynamic and flexible characteristics of the robotic arm mechanism in real-time operator work. Relative displacements of docking assemblies during their mating are minor. If a primary mechanical link is obtained before the alignment of their docking planes (before their stiff contact), a 6-DOF hybrid simulation is applied for ground testing. In this case, real assemblies, visual monitoring and aiming aids and robotic arm control handles are used, whereas a spatial flexible robotic arm is replaced by a math model as a corresponding system of motion equations integrated in real time. This ground testing method was first suggested and implemented at Rocket-Space Corporation Energia (RSC Energia).

This paper presents the 6-DOF schematic and describes the testing of the berthing of Mini-Research Module (MRM-1, Rassvet) [1] to the International Space Station (ISS) with the SSRMS (Space Station Remote Manipulator System).

\section{Operations performed by remotely controlled space manipulators}

The anthropomorphic manipulator SSRMS [2, 3] has been actively used for ISS building and operation. It has seven degrees of freedom (Figure 1,a), its kinematic schematic is fully symmetrical with respect to the central (elbow) joint, which enables manipulator's "stepping over" from one fixation place (base point) to another preserving its functionalities. Such base 
points are located in different places on the ISS external surface increasing functional accessibility. Operator work places for SSRMS control are located inside the USOS modules "Destiny" and "Cupola". The Japanese module of the ISS is served by a fixedly mounted on it remotely controlled robotic arm [4], that has the same kinematics as the Space Shuttle Remote Manipulator System (SRMS).

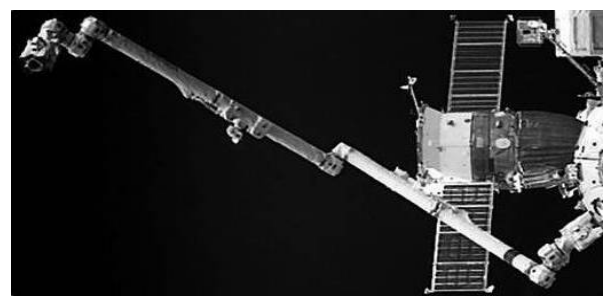

(a)

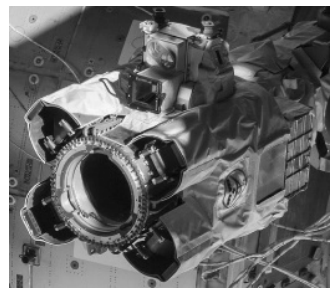

(b)

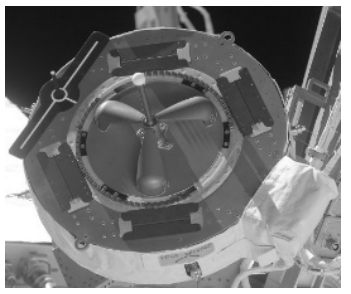

(c)

Figure 1. Remotely controlled ISS robotic arm SSRMS (a), an end effector with a TV camera (b) and a grapple fixture with a target (c) (NASA's picture)

Operations performed by remotely controlled space robotic arms basically consist of three stages: grappling the object, moving it, precise positioning with respect to the target device for approach and mating with it. At the first stage an SSRMS end effector (Figure 1,b) [5] is approaching a grapple fixture (Figure 1,c) [6] mounted on a mobile object. With the use of SRMS or SSRMS, mechanical mating is ensured by reaching the end of a grapple fixture with the end effector's tethers and their subsequent retraction. To obtain a precise guidance, a TV camera is used on the end effector and a target on the grapple fixture. The operator uses manual controls and visual information to control the process. An algorithm for solving the inverse manipulator's kinematic problem takes into account structural constraints and recalculates signals received from the manual controls to control signals for the servo drives.

Movement of the object to a target "assembly" location is performed automatically under computer control, as a rule, due to visual monitoring constraints. Moreover, this relieves the burden from the human operator. At the final stage, precise positioning or approach, the operator takes over again.

Remotely controlled space manipulators have significant linear dimensions (to move objects) and elastic flexibility of drive and link reducers. With their movement even small angular deformations may lead to significant lateral fluctuation of the end effector, which makes a precise positioning difficult. The drive power of robotic arms allows correct movement only with zero gravity. Therefore, it is practically impossible for the operator to obtain control skills on a real manipulator during ground training.

Taking into account relatively small dimensions of the end effector and grapple fixture, the process of grabbing an object on the ground can be quite accurately represented with the use of hybrid simulation; the dynamics of their relative movement is computed real-time and reproduced with a real industrial robotic arm. As the pin of the grapple fixture has a very simple geometry, it is possible to develop a software system for real-time simulation of a manipulator's movement and the fact of grabbing a grapple fixture (without its retraction by tethers).

Besides moving cargo items and assisting astronauts, robotic arms are used to perform a more complicated operation, i.e. berthing new modules of space transport vehicles to the ISS. To do this, the robotic arm has to grab an object and move it to a target location area. Mating 
of a module or a vehicle with the space station is performed with the use of universal docking [7] or simpler devices specially designed for berthing taking into account precise guidance with the robotic arm controlled by a human operator [8]. The latter option allows precise realtime math simulation of the interaction process [9].

For spacecraft berthing with docking assemblies, the main task of the operator controlling the robotic arm is to achieve capture, i.e. the latches of one assembly are clutched by the mechanical stops of the other and the robotic arm is relieved (drives and brakes in the joints switch off). After that, docking mechanisms provide their retraction and stiff connection. The robotic arm, remaining connected to the object, exerts a minor resistance.

The flexibility of the robotic arm of the robotic arm depends on its configuration. The grapple fixture used by the arm to grab an object is placed in the periphery of the target object. When a vehicle or a module, which normally has a significant mass, is moving, a reciprocal influence of their translational and angular motions occurs. Docking mechanisms ensuring capture have different flexibility, they are often used with a specific type of robotic arm for the first time. Taking into account these aspects, it may be necessary at the berthing design phase to estimate whether capture is possible to be achieved.

\section{Hybrid simulation of spacecraft berthing}

When designing spacecraft berthing with the use of a new mating device or docking assembly that was not originally targeted to work with a robotic arm, it is necessary to estimate in advance whether this operation is feasible to be implemented. Such an assessment can be made based on a hybrid simulation on the 6-DOF designed and developed at RSC Energia and used for ground testing of spacecraft docking assemblies. An adaptation of this facility to berthing testing is feasible, first of all because relative displacements of docking assemblies or devices are less when implementing berthing that docking. Figure 2 presents a simplified schematic of a 6-DOF dynamics facility for hybrid simulation.

A real-time simulation is performed with real docking assemblies (1), their relative displacements are reproduced by the 6-DOF platform (2) with Gough-Stewart kinematics [10, 11] and controllable hydraulic drives (3). One of the assemblies is mounted on the moving platform, the other on the device for measuring forces and moments of contact interaction (4) located on the load bearing structure (5) above the moving platform. This measuring device contains six strain gauge sensors oriented in non-parallel directions. Signals (6) from these sensors get to the input of the analog-digital convertor and then to the control computer (7) that performs several functions. Firstly, its software converts the signals from the six sensors to three components of force and three components of moment of docking assembly contact interaction. Secondly, it integrates differential equations of mating spacecraft relative motion dynamics, computes the values of relative positions and velocities of docking assemblies, and controls the 6-DOF moving platform. Initial conditions and mount points for each assembly (on the moving platform or on the device for measuring forces and moments) are defined in the test program. Inputs for motion math model are the geometrical and inertia properties of the mating spacecraft, measured values of forces and moments of contact interaction, and the readings of active docking mechanism sensors $(8)$. The values of forces and moments created by the control system of the active object are computed in the model. Thirdly, the control computer software converts relative positions and velocities of the docking assemblies to required linear displacements and velocities of hydraulic drive rods $(9)$ of the moving platform by solving its inverse kinematic problem. These control signals (10) get to the inputs of each hydraulic drive. 


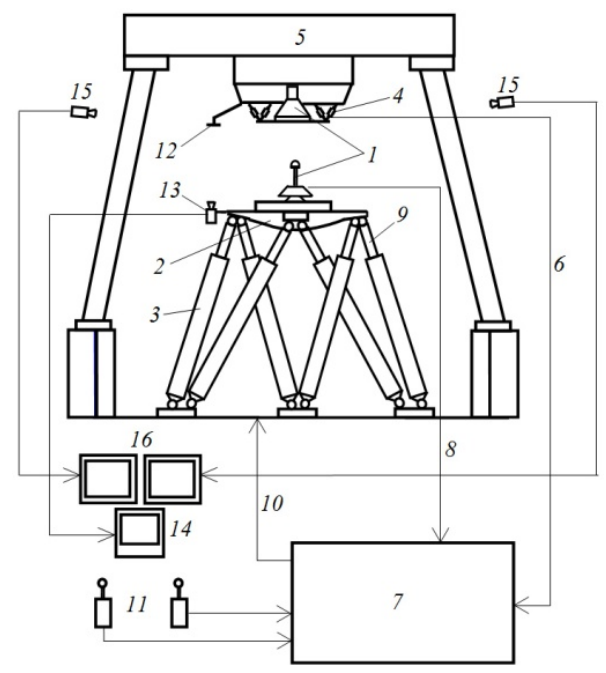

Figure 2. A simplified schematic of a 6-DOF berthing test facility

The hybrid simulation for each test case of ground berthing testing is implemented as follows. In advance, the moving platform is automatically, under software control, set to a specified position with a specified relative velocity that correspond to the initial conditions for the docking assemblies and shall be ensured by the robotic arm in real flight. After that, controlling is based on solving the system of differential equations of mating spacecraft relative motion, taking into account the initial conditions, measured forces and moments of contact interaction, as well as forces and moments of the active object control system that are computed from the readings of docking mechanism discrete sensors fixing the first contact and capture events. A docking process is not entirely simulated; it stops before a contact of docking planes of the assemblies occurs. This is explained by the fact that an increase of contact stiffness up to a value comparable with the axial stiffness of the moving platform hydraulic drives causes 'auto-oscillations' in the control loops of the hydraulic drives. Each test case is completed when a given maximal simulation time elapses or aborted if given maximal values of forces and moments of contact interaction are exceeded. To allow followup analysis, files with test results are generated. Besides measured values of forces and moments, recorded data contain relative positions and velocities of mating spacecraft and assemblies, readings of discrete and analog sensors of the docking mechanism.

To reproduce a spacecraft berthing on a 6-DOF facility, the motion model integrates differential equations and computes kinematic relations that describe displacements of these spacecraft as well as of the robotic arm carrying one of them. The human operator generates signals to the manipulator model. He normally uses two manual controls (11). The left manual control sets required linear velocities and the right one required angular velocities of the carried object. The reference frame in which these values are defined is linked to the target (12) mounted next to the assembly of berthing destination. The operator is watching the target with a TV camera (13) mounted on the carried spacecraft next to its docking assembly and with a monitor (14) on the operator work place. If a correction of the mating spacecraft relative angular position is needed, additional observation TV cameras (15) and monitors (16) can be used. 
Up to now, reals docking assemblies and a 6-DOF facility have been available only at RSC Energia. Partly due to this fact, Russia has a priority in such type of ground testing of spacecraft berthing.

\section{Ground testing of MRM-1 (Rassvet) module berthing to the ISS}

The design of MRM-1 module integration to the ISS FGB module was conducted from early 2007 to spring 2010. The MRM-1 module was assumed to be delivered with a Space Shuttle and carried, in the computer controlled mode, to its destination area by its on-board SRMS and the ISS manipulator SSRMS in the computer controlled mode. After that, the SSRMS operator was to provide capture of the two assemblies of the Russian 'probe-cone' docking system [7] (Figure 3).

Limit loads to robotic elements are significantly lower than those to docking assemblies. Therefore, high approach velocities are not acceptable. To recover misses and achieve capture with low velocities, the manipulator needs to create a force ultimate for its drive. The point of joint research of NASA Johnson Space Center, Canadian Space Agency (CSA) and RSCE Energia was a development of SSRMS control procedure that would allow fulfilling this task provided compliance with mutually acceptable limitations.

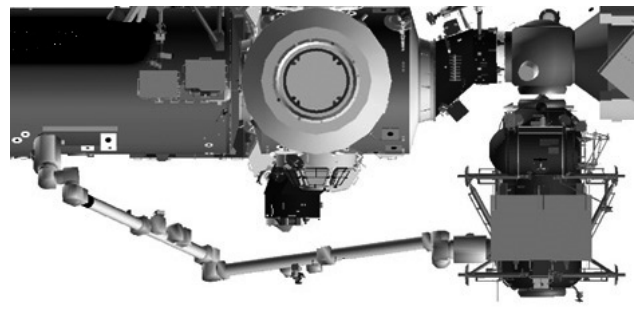

(a)

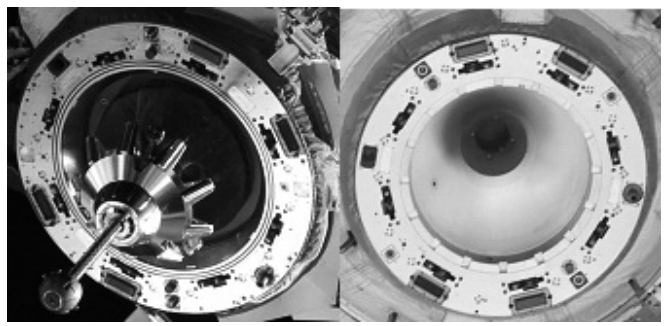

(b)

Figure 3. SSRMS configuration for MRM-1 (a) berthing (NASA data), active and passive assemblies of the 'probe-cone' docking system (b)

An aiming TV camera on the berthing module and a target on the ISS mounted near the passive assembly, the same as those used for a regular docking, were foreseen to be used for MRM-1 guidance during berthing.

The grapple fixture to be grabbed by the end effector is located at a certain distance from the center of mass of the carried module; its orientation is different from the approach direction. Due to this fact, the force of the flexible manipulator applied on the module causes its additional spinning even though commands are issued for translational displacements only. During guidance, first, angular misalignments between the axes of the docking assemblies were repeatedly compensated based on their images generated by the observation TV cameras, then a 'pure' lateral miss and angular misalignment with the lateral axis with the aiming TV camera. The art of control here was to minimize the number of such alignments.

For testing berthing on a 6-DOF, RSC Energia used robotic arm manual controls, TV cameras, target and monitor available on-site. Any hardware differences were assumed, and thereafter confirmed in practice, to be compensated by a human operator's adaptive capacity. The aiming TV camera was mounted on the moving platform, the target on the immobile part of the facility, the two observation TV cameras on the facility walls. Acquired images are presented in Figure 4. 


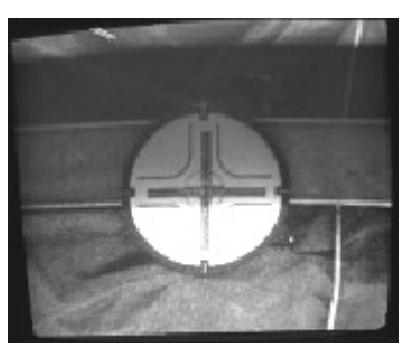

(a)

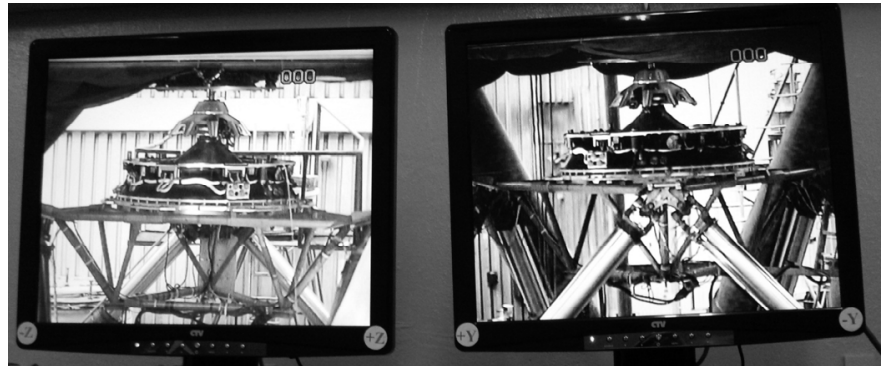

(b)

Figure 4. Images acquired from the aiming TV camera (a) and observation TV cameras (b) at hybrid simulation of MRM-1 berthing

Two manipulator control modes were simulated and analyzed upon capture acquisition. Real-time control was implemented by the operator. Based on imagery from the aiming and observation TV cameras, the operator set up required velocities of the module with two control handles. He also ensured a required alignment of docking assemblies. Time to capture acquisition could reach as much as 15 minutes.

A simplified control was performed without an operator. A maximum allowable approach velocity was set up; lateral and angular misalignments were evenly ranged depending on the capabilities of the docking assemblies.

For each hybrid simulation test case, the resulting file contained the issued manual control signals, velocities and rotation angles in the manipulator joints, kinematic parameters of the module, signals from discrete and analog sensors of the active docking assembly mechanism.

To ease the prompt analysis of a big variety of data, a visualization software is used to generate an integrated multi-window berthing representation called a Dynamic Diagram (Figure 5). Its upper window (Window 1) displays docking assembly contact interaction data: the frame model of the docking assemblies, kinematic parameters of their relative motion, signals from discrete and analogue sensors of the docking mechanism. Window 2 in the center of the diagram shows the configuration (angles in the joints and geometry) of the arm and directions of the axes of its joints, control signals acquired from the left (HL1-HL3) and right (HR1-HR3) handles. A mouse-scalable window on the right central part (Window 3) shows relative positions of the mating objects, docking assemblies, aiming TV camera and docking target. The lower window displays contact forces or other simulated parameters as functions of time. The vertical central line corresponds to current simulation time. The visualization program is linked to the file with simulated case results. When the file is continuously reviewed, the time functions in the lower window are moving from left to right passing through the current time axis. Their numerical values are displayed on the left side of the lower window. The contents of the upper and central windows of the dynamic diagram change with the current time.

Figure 5 and animation file accessible by link indicated in in Reference paper [12] Figure 9 caption, presents hybrid simulation results of a case without alignment, which takes shorter to run. At this case, capture is acquired on the $48^{\text {th }}$ second. The axial contact force, slightly exceeding $1000 \mathrm{~N}$, is mainly created when the probe head hits the bottom of the receiving cone socket as the manipulator does not stop at capture. 


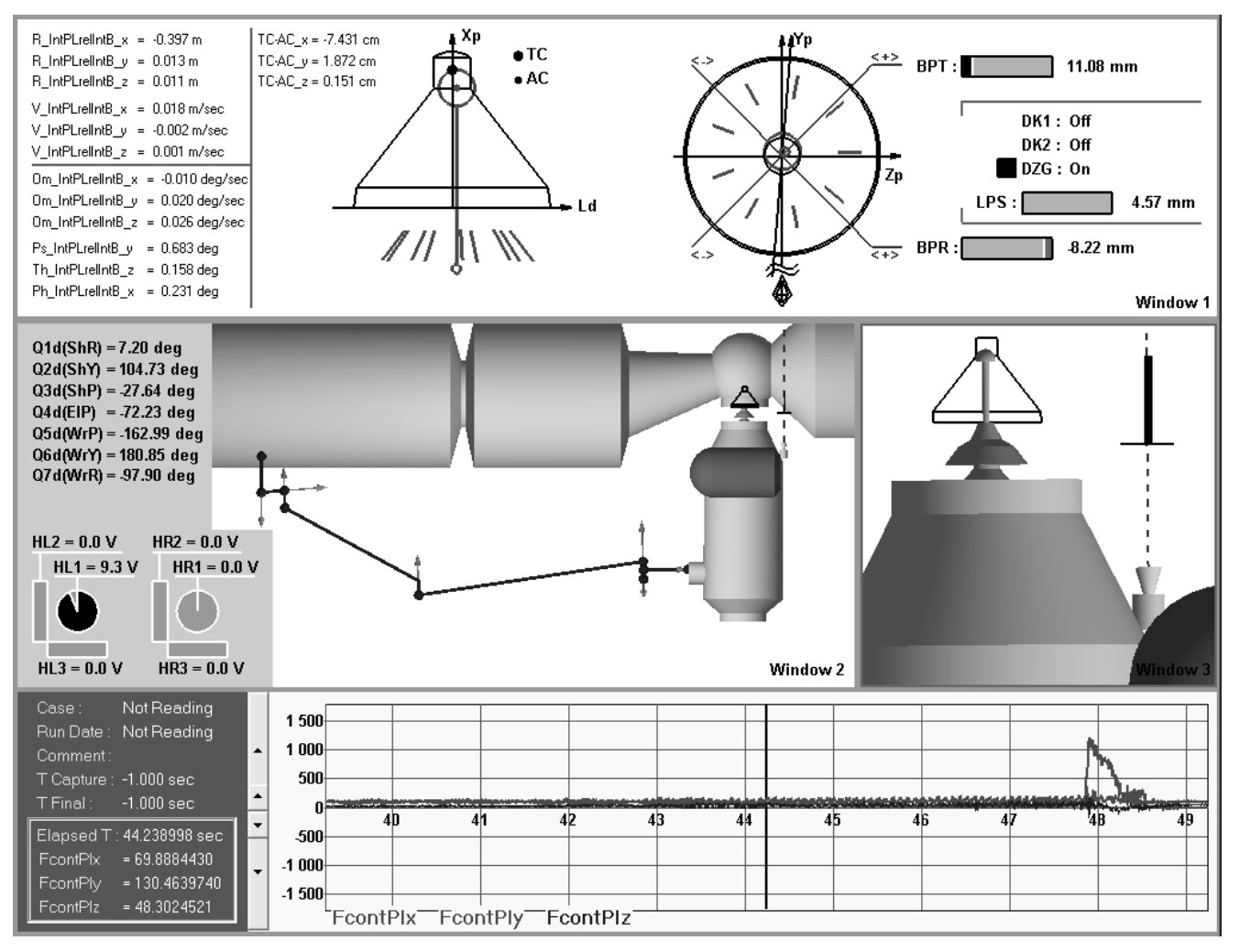

Figure 5. Dynamic diagram visualizing MRM-1 berthing (control without alignment)

At the case of precise guidance, the operator of the robotic arm first performed angular alignment using data from observation TV cameras, then compensated for a lateral miss based on the target image transmitted from the aiming TV camera. At the final stage, before the probe head hit the receiving cone socket, the operator mainly set up the approach velocity and performed a slight correction of lateral misses and angular misalignments. This enabled capture with an acceptable SSRMS load level.

6-DOF ground testing of MRM-1 berthing provided the following main results.

1. For precise control with a relative alignment of docking assemblies, capture is always acquired and contact loads do not exceed allowable limits for the arm.

2. For control without alignment, loads on the arm might exceed allowable limits, and capture might be impossible to occur.

3. The mail source of information for pitch and yaw angular alignments are imagery of docking assemblies acquired from the observation TV cameras. Subsequent compensations for lateral miss and roll are performed using the aiming TV camera.

4. MRM-1 (Rassvet) berthing to ISS by SSRMS was proved possible provided precise guidance (alignment) during approach.

The described berthing operation was successfully implemented on orbit on May 18, 2010. The acquired experience can be useful for designing space stations of a next generation.

\section{REFERENCES}

1. https://ru.wikipedia.org > wiki > Rassvet_(module_ISS). Accessed on 09.01.2020.

2. Canadaarm2's Data Sheet. URL: https://www.asc-csa.gc.ca/eng/iss/canadarm2/datasheet.asp. Accessed on 16.01.2020.

3. Mobile Servicing System. URL: https://en.wikipedia.org/wiki/Mobile_Servicing_System. 
4. Japanese Experiment Module Remote Manipulator System. URL: http://iss.jaxa.jp/en/kibo/about/kibo/rms/. Accessed on 16.01.2020.

5. https://commons.wikimedia.org/wiki/File:Canadarm2_Latching_End_Effector_ISS049E-004178).jpg. Accessed on 16.01.2020.

6. https://www.wikiwand.com/en/Grapple fixture. Accessed on 16.01.2020.

7. Syromyatnikov V.S. Docking Systems of Space Vehicles. M.: Mashinostroeniye, 1984. $216 \mathrm{c}$.

8. McLaughlin R.J., Warr W. H. The Common Berthing Mechanism (CBM) for International Space Station. https://spacecraft.ssl.umd.edu/design lib/ICES012435.ISS CBM.pdf. Accessed on 16.01.2020.

9. Ma O., Buhariwala K., Roger N., MacLean J., Carr R. MDSF - A Generic Development and Simulation Facility for Flexible, Complex Robotic Systems // Robotica, 1997. V. 15. P. 49-62.

10. Gough V. E., Whitehall S.G. Universal Tyre Test Machine // Proc. $9^{\text {th }}$ International Technical Congr. FISITA. London: Institution of mechanical Engineers, 1962. P. 117137.

11. Stewart D. A Platform with Six Degrees of Freedom // Proc. Institute of Mechanical Engineers. 1965. V. 180. Pt. 1, №. 15, P. 371-386.

12. Golubev Yu.F., Yaskevich A.V. Spacecraft berthing hybrid simulation on 6DOF dynamical facility // Preprints of Keldysh Institute of Applied Mathematics of Russian Academy of Sciences. 2019. № 117, $24 \quad$ P. doi:10.20948/prepr-2019-117, URL: http://library.keldysh.ru/preprint.asp?id=2019-117 